In etwas weniger als vier Jahren konnte der Verein SwissDRG nur einen Teil der Ziele erreichen, die er sich gesetzt hatte. Er wurde durch die ideologischen Kämpfe unter seinen Mitgliedern gebremst und musste viel Energie aufwenden, um Verzögerungsmanövern zu begegnen. Nachdem die politischen Fragen nun geklärt sind, ist mit der SwissDRG AG auf einen gewissen Neuanfang zu hoffen, da alle Projektpartner eine dynamische Tarifstruktur befürworten. In der Praxis steht dem CMO eine enorme Aufgabe bevor, da es verschiedene nicht abgeschlossene Baustellen des Vereins übernimmt und zugleich seine zentrale Rolle in der Leitung eines Systems wahrnehmen muss, das sich mitten im Aufbau befindet. Das CMO wird auf die Unterstützung des Verwaltungsrats angewiesen sein, dessen Mitglieder sich alle als loyal erweisen sollten. Obwohl es der Ärzteschaft gelungen ist, im neuen Unternehmen einen Platz zu erhalten, müssen wir wachsam bleiben. Dies gilt vor allem für Bereiche wie die Qualitätskriterien oder den Datenschutz, in denen uns eine zentrale Rolle zukommt.

Unsere Partner wissen, dass die Zusammenarbeit mit den Ärztinnen und Ärzten unabdingbar ist, und erwarten von uns ein hohes Engagement, damit ein leistungsfähiges System erreicht werden kann. Wir haben unsere Fähigkeiten unter Beweis gestellt und belegt, dass wir in der Lage sind, geschlossen aufzutreten und unter Zeitdruck ein grosses Arbeitspensum zu bewältigen. Die Glaubwürdigkeit, die wir damit im Helvetisierungsprozess gewonnen haben, muss nun weiter gepflegt werden. Die FMH ist bereit, diese Herausforderung durch eine minutiöse Planung und den schrittweisen Einsatz der notwendigen Mittel im Verlauf der kommenden Jahre anzunehmen.

Dr. med. Pierre-François Cuénoud,

Mitglied des Zentralvorstands, Leiter Ressort SwissDRG

\title{
Gründung der SwissDRG AG: Wird endlich gut, was lange währt?
}

Nach mehrmaligen Verzögerungen ist es nun soweit: Die Aktionäre $\mathrm{H}+$, die FMH, santésuisse und die Gesundheitsdirektorenkonferenz GDK haben am 18. Januar 2008 die SwissDRG Aktiengesellschaft mit Sitz in Bern gegründet. Der Verein und das Projekt SwissDRG wurden damit in eine gemeinnützige Aktiengesellschaft überführt. Die SwissDRG AG bzw. das Case Mix Office (CMO) als operatives Organ soll die Einführung des schweizweit einheitlichen Fallpauschalensystems im akutstationären Bereich sowie die Pflege der Tarifstruktur gewährleisten. Gemäss Beschluss der eidgenössischen Räte hat die flächendeckende Einführung der Fallpauschalen bis spätestens Ende 2011 zu erfolgen.

Mit der Gründung der Aktiengesellschaft hat Swiss DRG einen wichtigen Meilenstein erreicht. Und was hat die Ärzteschaft in diesem Prozess bisher bewirkt? Erreicht hat die Ärzteschaft nicht nur, dass die FMH einen Sitz im Verwaltungsrat der SwissDRG AG innehat. Erreicht hat die Ärzteschaft auch, dass die Helvetisierung, d.h. die Anpassung des deutschen Systems an die Schweizer Verhältnisse, unter Einbezug der Fachgesellschaften erfolgt. Dies, nachdem die Projektpartner das SwissDRG-Vergütungssystem anfänglich - wie beim deutschen G-DRG und beim Schweizer APDRG - ohne die Fachgesellschaften erarbeiten wollten. Die FMH-Arbeitsgruppe Helvetisierung und die Fachgesellschaften haben trotz des grossen Zeitdrucks in der ersten Helvetisierungsrunde (2007) bewiesen, dass der Einbezug ihres ärztlichen Fachwissens für den Aufbau eines Vergütungssystems unentbehrlich ist. Die FMH ist somit aktiv an der Ausgestaltung der Tarifstruktur beteiligt. Bedauerlich ist allerdings, dass die FMH nicht als Tarifpartner anerkannt und damit nicht in die Tarifverhandlungen zur Bestimmung der Base Rate eingebunden wird.

Damit das SwissDRG-System ein möglichst leistungsgerechtes System wird, gibt es noch viel zu tun. Einerseits gilt es, das System weiter an die Schweizer Verhältnisse anzupassen. Sobald die neue Grouperversion zur Verfügung steht, werden die Fachgesellschaften wieder gefragt sein, ihre Anträge zur Verbesserung des Systems via FMH einzureichen. Andererseits müssen für verschiedene übergeordnete Problempunkte endlich Lösungen gefunden werden. Beispielsweise soll auch unter SwissDRG für die Spitäler ein Anreiz bestehen, die erforderliche Ausund Weiterbildung zu betreiben und innovative Verfahren zu fördern. Zudem ist zu verhindern, dass Kinderspitäler systembedingt ums Überleben kämpfen müssen. Auch die Vergütung der Belegärzte ist zu klären. Weiter muss die Versorgungsqualität ebenfalls unter dem neuen Vergütungssystem sichergestellt werden, steht doch immer noch das Wohl der Patienten im Zentrum. Zentral ist es, dass vor der Einführung von SwissDRG eine Begleitforschung aufgebaut wird, um Fehlsteuerungen frühzeitig zu erkennen.

Wertvolle Zeit ist durch die Verzögerung der Gründung der SwissDRG AG verstrichen. Das CMO muss die verbleibende Zeit nun optimal nutzen, die «heissen Eisen» endlich anpacken und die Lösungsfindung für die anstehenden Problempunkte vorantreiben.

Beatrix Meyer,

operative Leiterin SwissDRG der FMH 